Bundesgesundheitsbl 2013 · 56:678-686

DOI 10.1007/s00103-013-1687-4

Online publiziert: 27. Mai 2013

(c) Springer-Verlag Berlin Heidelberg 2013

\section{Additional material online}

An English full-text version of this article is available at SpringerLink under supplementary material: dx.doi.org/10.1007/s00103-013-1687-4

\section{Hintergrund und Fragestellung}

Zu den häufigsten chronischen Erkrankungen zählen entzündliche und degenerative Erkrankungen des Bewegungsapparats, die unter dem Begriff muskuloskelettale Erkrankungen (MSKE) zusammengefasst werden. MSKE betreffen einen Großteil der älteren und alten Bevölkerung und sind weltweit die führende Ursache von chronischen Schmerzen, körperlichen Funktionseinschränkungen und Verlust an Lebensqualität $[1,2,3]$.

Nach Schätzungen der Weltgesundheitsorganisation (WHO) wird sich die Anzahl der von Knochen- und Gelenkerkrankungen betroffenen Personen in den Jahren 2000 bis 2020 verdoppelt haben [4]. International werden daher vielfach Anstrengungen unternommen, muskuloskelettalen Erkrankungen und der damit verbundenen Krankheitslast gerecht $\mathrm{zu}$ werden. So rief die WHO beispielsweise die „Bone and Joint Decade 20002010“ aus, um die Forschungs- und Versorgungssituation zu verbessern.

MSKE gehören auch in Deutschland zu den häufigsten Leiden [5]. Sie verursachen durch Aufwendungen für krankheitsspezifische Behandlungen und infolge von Arbeitsunfähigkeit und vorzeitiger Berentung hohe volkswirtschaftliche Kosten $[6,7]$.

Bevölkerungsrepräsentative Daten zur gegenwärtigen Verbreitung von MSKE

J. Fuchs - M. Rabenberg • C. Scheidt-Nave

Abteilung für Epidemiologie und Gesundheitsmonitoring, Robert Koch-Institut, Berlin

\title{
Prävalenz ausgewählter muskuloskelettaler Erkrankungen
}

\section{Ergebnisse der Studie zur Gesundheit Erwachsener in Deutschland (DEGS1)}

in Deutschland sind größtenteils krankheitsspezifisch und stützen sich meist auf kleinräumige Datenerhebungen oder Diagnose- und andere Routinedaten [7, 8, 9, 10, 11, 12, 13, 14, 15, 16, 17]. Zu den MSKE zählen sowohl degenerative Gelenkerkrankungen (z. B. Arthrose), entzündliche Gelenkerkrankungen (z. B. rheumatoide Arthritis) und Skeletterkrankungen (z. B. Osteoporose). Im Folgenden wird für die 3 genannten Erkrankungen die aktuelle Verbreitung für die in Deutschland lebende Bevölkerung beschrieben.

\section{Arthrose}

Arthrose ist die weltweit häufigste Gelenkerkrankung $[4,18]$ und verursacht mehr Einschränkungen und Behinderungen in der älteren Bevölkerung als jede andere Erkrankung [3]. Charakteristisch für die Arthrose sind degenerative Veränderungen an den Gelenken. Diese beginnen mit der sukzessiven Zerstörung des Gelenkknorpels und können bis zur Freilegung der Knochenoberfläche führen. Auch die Schädigung angrenzender Strukturen wie Knochen, Muskeln und Bänder sind häufig zu beobachten. Arthrose kann durch pathologische Veränderungen im Röntgenbild diagnostiziert werden, aber auch durch Gelenkbeschwerden. Als unveränderbare Risikofaktoren gelten zunehmendes Alter, weibliches Geschlecht und genetische Veranlagung [19, 20, 21]. Hinzu kommen erworbene Ursachen oder Mitursachen wie Über- und Fehlbelastung der Gelenke bei angeborenen Deformitäten (z. B. Achsenfehlstellungen, Hüftgelenkdysplasien), nach Verletzungen und Unfällen oder durch Übergewicht [22].

\section{Rheumatoide Arthritis (RA)}

Unter dem Begriff Arthritis werden chronisch entzündliche, systemische Gelenkerkrankungen zusammengefasst, die auf der Grundlage bisher nicht vollständig geklärter Autoimmunprozesse entstehen. $\mathrm{Zu}$ den wichtigsten Risikofaktoren für diese chronisch entzündlichen $\mathrm{Ge}$ lenkerkrankungen zählen weibliches Geschlecht, höheres Lebensalter, Umweltfaktoren wie Rauchen und eine Vielzahl genetischer Faktoren [23, 24, 25].

Entzündliche Gelenkerkrankungen verlaufen meist schubweise und fortschreitend. Sie ziehen häufig chronische Funktionsbeeinträchtigungen nach sich, verbunden mit Schmerzen und Einschränkungen der Lebensqualität. Die häufigste entzündliche Gelenkerkrankung ist die rheumatoide Arthritis (RA) $[3,26]$. Die RA ist klinisch charakterisiert durch Gelenkschwellung und Schmerzen und führt zu erosiver Gelenkdestruktion und Funktionsbehinderung [27]. Die Diagnose stützt sich auf Klassifikationskriterien, die 2010 mit dem Ziel von verbesserter Frühdiagnostik und Frühtherapie ak- 
tualisiert wurden [28]. Die Kriterien beziehen sich auf die Zahl betroffener Gelenke, die Serologie, Entzündungsmarker und die Symptomdauer.

\section{Osteoporose}

Bei der Osteoporose handelt es sich um eine systemische Skeletterkrankung, die durch einen Abbau an Knochenmasse und eine Verschlechterung der mikroarchitektonischen Knochenstruktur charakterisiert ist [29], was eine geringe Bruchfestigkeit des Knochens zur Folge hat [30]. Dadurch kommt es schon bei geringfügigen äußeren Anlässen zu Knochenbrüchen (Fragilitätsfrakturen). Typische Lokalisationen für Fragilitätsfrakturen sind in erster Linie die Wirbelkörper, die hüftgelenknahen Abschnitte des Oberschenkelknochens (Femurhals und Trochanter-Region) sowie der handgelenknahe Abschnitt der Speiche (distaler Radius).

Zur Entstehung von Osteoporose trägt eine Vielzahl von Faktoren bei. Dazu gehören nicht modifizierbare Faktoren wie zunehmendes Alter, weibliches Geschlecht und familiäre Veranlagung sowie verhaltensbasierte, beeinflussbare Risikofaktoren wie Bewegungsmangel und Fehlernährung, Grunderkrankungen und $\mathrm{Me}$ dikationen, die ursächlich behandelt oder angepasst werden können [29].

Besondere gesundheitspolitische Bedeutung erhält die Osteoporose durch ihre enge Kopplung an das höhere Lebensalter und die Frakturfolgen. Gerade hüftgelenknahe Frakturen und Wirbelkörperfrakturen führen zu Einbußen an Lebensqualität und selbstständiger Lebensführung. Da hüftgelenknahe Frakturen im Gegensatz zu Wirbelkörperfrakturen immer diagnostiziert und operativ versorgt werden, verursachen sie die höchsten direkten Behandlungskosten [31, 32].

\section{Methoden}

\section{Studiendesign und Stichprobe}

Die „Studie zur Gesundheit Erwachsener in Deutschland" (DEGS) ist Bestandteil des Gesundheitsmonitorings des Robert Koch-Instituts (RKI). Konzept und Design von DEGS sind an anderer Stel- le ausführlich beschrieben $[33,34,35,36$, 37]. Die erste Erhebungswelle (DEGS1) wurde von 2008 bis 2011 durchgeführt und umfasste Befragungen, Untersuchungen und Tests $[38,39]$. Zielpopulation war die in Deutschland lebende Bevölkerung im Alter von 18 bis 79 Jahren. DEGS1 hat ein Mischdesign, das gleichzeitig quer- und längsschnittliche Analysen ermöglicht. Hierbei wurde eine Einwohnermeldeamtsstichprobe gezogen, die ehemalige Teilnehmerinnen und Teilnehmer des Bundes-Gesundheitssurveys 1998 (BGS98) ergänzt. Insgesamt nahmen 8152 Personen teil, darunter 4193 Ersteingeladene (Response 42\%) und 3959 ehemalige Teilnehmerinnen und Teilnehmer des BGS98 (Response 62\%). 7238 Personen besuchten eines der 180 Untersuchungszentren, 914 wurden ausschließlich befragt. Die Nettostichprobe [34] ermöglicht für den Altersbereich von 18 bis 79 Jahren repräsentative Querschnittanalysen und Trendaussagen im Vergleich mit dem BGS98 $(n=7988$, davon 7116 in Untersuchungszentren). Die Daten der erneut Teilnehmenden sind für Längsschnittanalysen nutzbar. Eine Nonresponder-Analyse und der Vergleich einzelner erhobener Indikatoren mit Daten der amtlichen Statistik weisen auf eine hohe Repräsentativität der Stichprobe für die Wohnbevölkerung in Deutschland hin [34].

\section{Variablen}

Die Angaben zu den Lebenszeitprävalenzen, also dem Auftreten der Krankheit in der gesamten Lebensdauer, von Arthrose, rheumatoider Arthritis und Osteoporose erfolgen auf Basis von Selbstangaben zu ärztlich diagnostizierten Erkrankungen während eines computergestützten ärztlichen Interviews. Osteoporose wird aufgrund der bekannten, sehr niedrigen Prävalenzen im jungen und mittleren Erwachsenenalter nur bei Befragten ab dem Alter von 50 Jahren erfasst.

Die Teilnehmenden wurden gefragt, ob bei ihnen von einem Arzt jemals die entsprechende Krankheit festgestellt wurde und falls ja, wann diese diagnostiziert wurde, bei welchem Arzt (Hausarzt, Facharzt) sie deswegen in ärztlicher Behandlung sind und welche Art der Behand- lung (z. B. Medikamente, Krankengymnastik) sie erhalten. Bei Nachfragen gaben die Ärzte standardisierte Hinweise zu den einzelnen Erkrankungen. So wurde z. B. bei der RA auf Nachfragen erläutert, dass damit eine schwere entzündliche Gelenkerkrankung, die meist beidseitig auftritt und fast immer die Fingergelenke mit einschließt, gemeint ist. Personen, die eine ärztlich diagnostizierte Arthrose angegeben hatten, wurden gebeten anzugeben, an welchem Gelenk bzw. welchen Gelenken die Arthrose festgestellt wurde.

Bei allen 3 Erkrankungen werden die Lebenszeitprävalenzen insgesamt sowie differenziert nach Alter, Geschlecht, Sozialstatus und Region im Querschnitt ermittelt.

Der Sozialstatus wurde anhand eines Indexes bestimmt, in den Angaben zu schulischer und beruflicher Ausbildung, beruflicher Stellung sowie Haushaltsnettoeinkommen (bedarfsgewichtet) eingehen und der eine Einteilung in niedrige, mittlere und hohe Statusgruppen ermöglicht [40].

\section{Statistische Auswertung}

Die Ergebnisse zu den einzelnen MSKE wurden mit 95\%-Konfidenzintervallen (95\%-KI) nach Geschlecht und Altersgruppe unter Einbeziehung des Sozialstatus berechnet. Personen mit fehlenden Angaben wurden von den Analysen ausgeschlossen.

Die Querschnittanalysen werden mit einem Gewichtungsfaktor durchgeführt, der Abweichungen der Stichprobe von der Bevölkerungsstruktur (Stand 31.12.2010) hinsichtlich Alter, Geschlecht, Region und Staatsangehörigkeit sowie Gemeindetyp und Bildung korrigiert [34]. Für den Untersuchungsteil wurde ein gesonderter Gewichtungsfaktor erstellt. Bei der Berechnung der Gewichtung für die ehemaligen Teilnehmenden des BGS98 wurde die Wiederteilnahmewahrscheinlichkeit, basierend auf einem logistischen Modell, berücksichtigt.

Um sowohl die Gewichtung als auch die Korrelation der Teilnehmenden innerhalb einer Gemeinde zu berücksichtigen, wurden die Konfidenzintervalle mit den Verfahren für komplexe Stichproben von SPSS-20 bestimmt. Unterschiede werden 
als statistisch signifikant angesehen, wenn sich die jeweiligen 95\%-Konfidenzintervalle nicht überschneiden oder in logistischen Regressionsanalysen die p-Werte kleiner als 0,05 sind.

\section{Ergebnisse}

\section{Arthrose}

Eine Übersicht über die Ergebnisse zur Lebenszeitprävalenz der Arthrose, gegliedert nach Geschlecht, Altersgruppe und Sozialstatus, findet sich in • Tab. 1.20,2\% aller Befragten zwischen 18 und 79 Jahren geben an, jemals eine ärztlich diagnostizierte Arthrose gehabt zu haben. Frauen sind dabei signifikant häufiger (22,3\%) von Arthrose betroffen als Männer (18,1\%). Der Anteil von Personen, der angibt, eine ärztlich diagnostizierte Arthrose zu haben, steigt mit zunehmendem Alter sowohl bei Frauen als auch bei Männern signifikant an. Demnach sind in der Altersgruppe der 18- bis 29-jährigen Frauen 1,6\% betroffen, bei den 70- bis 79-jährigen Frauen 49,9\%. Bei den Männern steigt die Prävalenz in den entsprechenden Altersgruppen von 1,8 auf $33,3 \%$.

Weder der Sozialstatus noch die Größe der Wohnortgemeinde (ländlich, kleinstädtisch, mittelstädtisch, großstädtisch) stehen in einem Zusammenhang mit dem Auftreten einer Arthrose. Bei Männern zeigen sich allerdings regionale Einflüsse: in den Regionen Nordrhein-Westfalen und Ost (neue Bundesländer und Berlin) geben weniger Männer eine Arthrose an als in den südlichen Regionen (Bayern, Baden-Württemberg) und Nordwest/Mitte (Schleswig-Holstein, Bremen, Hamburg, Niedersachsen, Hessen, Rheinland-Pfalz, Saarland).

Zur Lokalisation der Arthrose zeigt sich in - Abb. 1, dass bei beiden Geschlechtern bei mehr als der Hälfte der Befragten das Knie betroffen ist, jeweils etwa ein Viertel gibt eine Arthrose in der Hüfte an. Signifikant mehr Frauen als Männer geben eine Arthrose an den Fingergelenken an $(36,6 \%$ vs. $15,7 \%)$. Von der Hälfte der Personen mit Arthrose werden darüber hinaus andere betroffene Gelenke ohne weitere Lokalisation angegeben.

Bundesgesundheitsbl 2013 · 56:678-686

(c) Springer-Verlag Berlin Heidelberg 2013

\section{J. Fuchs $\cdot$ M. Rabenberg $\cdot$ C. Scheidt-Nave \\ Prävalenz ausgewählter muskuloskelettaler Erkrankungen. Ergebnisse der Studie zur Gesundheit Erwachsener in Deutschland (DEGS1)}

\section{Zusammenfassung}

Der Begriff muskuloskelettale Erkrankungen (MSKE) umfasst unter anderen entzündliche und degenerative Erkrankungen des Bewegungsapparats. MSKE sind weltweit häufig bei Älteren und stellen die führende Ursache von chronischen Schmerzen, körperlichen Funktionseinschränkungen und Verlust an Lebensqualität dar. In der Studie zur Gesundheit Erwachsener in Deutschland (DEGS1) wird die aktuelle Verbreitung der häufigsten MSKE Arthrose, rheumatoide Arthritis (RA) und Osteoporose in Deutschland erfasst. Die Auswertungen basieren auf den Angaben von 7988 Personen im Alter von 18 bis 79 Jahren (Osteoporose ab 50 Jahren), die in einem persönlichen Interview angaben, ob die jeweilige Erkrankung bei innen jemals ärztlich diagnostiziert wurde. Arthro- se liegt bei 22,3\% der Frauen und 18,1\% der Männer vor, RA bei 3,2\% der Frauen und 1,9\% der Männer. 13,1\% der Frauen und 3,2\% der Männer geben eine Osteoporose an. Bei allen MSKE sind Frauen signifikant häufiger betroffen als Männer. Die Schätzungen für beide Geschlechter steigen mit zunehmendem Alter an. MSKE spielen daher für die ältere und alte Bevölkerung in Deutschland eine bedeutende Rolle. DEGS1 bietet eine Vielfalt von Möglichkeiten, MSKE in Zusammenhang mit relevanten Einflussgrößen zu setzen und somit den Gesundheitszustand der zu Hause lebenden Bevölkerung präzise zu beschreiben.

Schlüsselwörter

Prävalenz · Arthrose - Rheumatoide Arthritis . Osteoporose · Gesundheitssurvey

\section{Prevalence of selected musculoskeletal conditions in Germany. Results of the German Health Interview and Examination Survey for Adults (DEGS1)}

\section{Abstract}

The term musculoskeletal condition (MSKC) comprises inflammatory and degenerative diseases of joints and bones. They are among the most common conditions in older age and cause of severe long-term pain, physical disability, and decrease in quality of life. Data from the German Health Interview and Examination Survey for Adults (DEGS1) were used to estimate the life-time prevalence of osteoarthritis, rheumatoid arthritis (RA) and osteoporosis in Germany. A total of 7,988 persons aged $18-79$ years (osteoporosis $50-79$ years) were asked to report doctor-diagnosed MSKC in face-to-face interviews. Women were more likely to report all MSKC and all prevalences increase with age. Osteoarthritis is reported by $22.3 \%$ of women and $18.1 \%$ of men, RA by $3.2 \%$ of women and $1.9 \%$ of men, and osteoporosis by $13.1 \%$ of women and $3.2 \%$ of men. MSKC are of great relevance for older adults in Germany. Data from DEGS1 provide a lot of information along to MSKC and hereby allow a closer description of the health situation of older adults. An English full-text version of this article is available at SpringerLink as supplemental.

\section{Keywords}

Prevalence - Osteoarthritis $\cdot$ Rheumatoid arthritis · Osteoporosis · Health survey

\section{Rheumatoide Arthritis (RA)}

Eine Übersicht über die Ergebnisse zur Lebenszeitprävalenz der RA, aufgegliedert nach Geschlecht, Altersgruppe und Sozialstatus, findet sich in $\bullet$ Tab. 2. Eine ärztlich diagnostizierte RA wird von 2,5\% aller 18- bis 79-Jährigen angegeben. Frauen weisen mit 3,2\% signifikant häufiger eine RA auf als Männer mit 1,9\%. Der Anteil von Personen mit RA nimmt bei beiden Geschlechtern mit dem Alter signifikant zu. In der jüngsten Altersgruppe liegt die Prävalenz mit $1,7 \%$ bei Frauen und 0,5\% bei Männern niedrig. Sie steigt bis zur Altersgruppe der 60bis 69-Jährigen bei beiden Geschlechtern stetig an, wobei Frauen mit 4,9\% eine deutlich höhere Prävalenz als Männer mit 2,9\% aufweisen. In der höchsten Altersgruppe der 70- bis 79-Jährigen zeigt sich bei Frauen eine unveränderte 
Tab. 1 Lebenszeitprävalenz der Arthrose nach Geschlecht, Altersgruppe und sozialem Status

\begin{tabular}{|c|c|c|c|c|c|c|c|c|c|c|}
\hline & & \multicolumn{7}{|c|}{ Altersgruppe } & \multicolumn{2}{|c|}{$\begin{array}{l}\text { Ungewichtete Fall- } \\
\text { zahlen, Arthrose }\end{array}$} \\
\hline & & $\begin{array}{l}18 \text { bis } \\
29 \text { Jahre }\end{array}$ & $\begin{array}{l}30 \text { bis } \\
39 \text { Jahre }\end{array}$ & $\begin{array}{l}40 \text { bis } \\
49 \text { Jahre }\end{array}$ & $\begin{array}{l}50 \text { bis } \\
59 \text { Jahre }\end{array}$ & $\begin{array}{l}60 \text { bis } \\
69 \text { Jahre }\end{array}$ & $\begin{array}{l}70 \text { bis } \\
79 \text { Jahre }\end{array}$ & Gesamt & Ja & Nein \\
\hline \multicolumn{11}{|l|}{ Frauen } \\
\hline \multirow[t]{8}{*}{ Sozialstatus } & Niedrig & $0,7 \%$ & $3,7 \%$ & $8,7 \%$ & $31,1 \%$ & $49,0 \%$ & $46,3 \%$ & $24,1 \%$ & 170 & 440 \\
\hline & $95 \%-K I$ & $0,1-4,6 \%$ & $1,2-11,2 \%$ & $4,7-15,8 \%$ & $21,3-42,9 \%$ & $38,9-59,3 \%$ & $36,7-56,1 \%$ & $20,4-28,3 \%$ & & \\
\hline & Mittel & $2,0 \%$ & $3,6 \%$ & $14,1 \%$ & $29,9 \%$ & $47,3 \%$ & $49,5 \%$ & $22,7 \%$ & 637 & 1814 \\
\hline & $95 \%-\mathrm{KI}$ & $0,8-4,6 \%$ & $1,7-7,1 \%$ & $10,6-18,5 \%$ & $25,4-34,7 \%$ & $41,8-52,9 \%$ & $43,0-56,1 \%$ & $20,6-24,9 \%$ & & \\
\hline & Hoch & $1,8 \%$ & $3,7 \%$ & $10,5 \%$ & $29,1 \%$ & $42,2 \%$ & $67,7 \%$ & $19,0 \%$ & 211 & 660 \\
\hline & 95\%-KI & $0,4-7,0 \%$ & $1,8-7,2 \%$ & $6,4-17,0 \%$ & $22,1-37,2 \%$ & $32,8-52,3 \%$ & $54,3-78,7 \%$ & $16,3-22,0 \%$ & & \\
\hline & Gesamt & $1,6 \%$ & $3,7 \%$ & $12,6 \%$ & $29,9 \%$ & $46,9 \%$ & $49,9 \%$ & $22,3 \%$ & 1018 & 2914 \\
\hline & (95\%-KI) & $0,8-3,2 \%$ & $2,3-6,1 \%$ & $10,1-15,7 \%$ & $26,6-33,5 \%$ & $42,6-51,2 \%$ & $45,2-54,5 \%$ & $20,8-23,8 \%$ & & \\
\hline \multicolumn{11}{|l|}{ Männer } \\
\hline \multirow[t]{8}{*}{ Sozialstatus } & Niedrig & $5,5 \%$ & $4,5 \%$ & $17,0 \%$ & $32,0 \%$ & $34,7 \%$ & $24,7 \%$ & $18,7 \%$ & 117 & 434 \\
\hline & 95\%-KI & $2,4-12,3 \%$ & $1,1-16,2 \%$ & $10,0-27,5 \%$ & $23,3-42,3 \%$ & $23,8-47,6 \%$ & $16,2-35,8 \%$ & $15,1-22,9 \%$ & & \\
\hline & Mittel & $0,5 \%$ & $10,8 \%$ & $14,3 \%$ & $28,9 \%$ & $33,5 \%$ & $36,6 \%$ & $18,6 \%$ & 411 & 1611 \\
\hline & 95\%-KI & $0,1-2,0 \%$ & $6,7-17,1 \%$ & $10,7-18,9 \%$ & $23,8-34,5 \%$ & $27,9-39,7 \%$ & $30,4-43,3 \%$ & $16,6-20,7 \%$ & & \\
\hline & Hoch & $1,8 \%$ & $11,0 \%$ & $9,0 \%$ & $20,7 \%$ & $33,0 \%$ & $33,8 \%$ & $16,6 \%$ & 187 & 802 \\
\hline & 95\%-KI & $0,3-11,7 \%$ & $5,8-20,1 \%$ & $5,5-14,3 \%$ & $14,2-29,1 \%$ & $25,6-41,3 \%$ & $24,8-44,2 \%$ & $13,7-19,9 \%$ & & \\
\hline & Gesamt & $1,8 \%$ & $9,6 \%$ & $13,2 \%$ & $27,5 \%$ & $33,8 \%$ & $33,3 \%$ & $18,1 \%$ & 715 & 2847 \\
\hline & 95\%-KI & $0,9-3,5 \%$ & $6,4-14,0 \%$ & $10,5-16,4 \%$ & $23,9-31,5 \%$ & $29,5-38,4 \%$ & $28,8-38,2 \%$ & $16,6-19,7 \%$ & & \\
\hline \multicolumn{11}{|l|}{ Gesamt } \\
\hline \multirow[t]{8}{*}{ Sozialstatus } & Niedrig & $3,0 \%$ & $4,2 \%$ & $13,2 \%$ & $31,6 \%$ & $42,5 \%$ & $38,7 \%$ & $21,5 \%$ & 287 & 874 \\
\hline & 95\%-KI & $1,4-6,5 \%$ & $1,6-10,4 \%$ & $8,8-19,4 \%$ & $25,0-39,0 \%$ & $35,1-50,2 \%$ & $31,0-47,0 \%$ & $18,7-24,5 \%$ & & \\
\hline & Mittel & $1,2 \%$ & $7,1 \%$ & $14,2 \%$ & $29,4 \%$ & $41,1 \%$ & $43,6 \%$ & $20,7 \%$ & 1048 & 3425 \\
\hline & 95\%-KI & $0,6-2,5 \%$ & $4,7-10,6 \%$ & $11,7-17,2 \%$ & $26,0-33,1 \%$ & $36,9-45,4 \%$ & $39,0-48,3 \%$ & $19,3-22,2 \%$ & & \\
\hline & Hoch & $1,8 \%$ & $7,6 \%$ & $9,7 \%$ & $24,8 \%$ & $36,4 \%$ & $45,8 \%$ & $17,6 \%$ & 398 & 1462 \\
\hline & 95\%-KI & $0,5-5,9 \%$ & $4,6-12,2 \%$ & $6,7-13,6 \%$ & $20,1-30,2 \%$ & $30,3-43,0 \%$ & $37,2-54,7 \%$ & $15,6-19,9 \%$ & & \\
\hline & Gesamt & $1,7 \%$ & $6,7 \%$ & $12,9 \%$ & $28,7 \%$ & $40,4 \%$ & $42,4 \%$ & $20,2 \%$ & 1733 & 5761 \\
\hline & $95 \%-\mathrm{KI}$ & $1,0-2,7 \%$ & $4,9-9,2 \%$ & $11,1-15,0 \%$ & $26,3-31,3 \%$ & $37,4-43,6 \%$ & $38,8-45,9 \%$ & $19,2-21,2 \%$ & & \\
\hline
\end{tabular}

Prävalenzrate von 4,9\%, während sie bei Männern auf 5,8\% steigt.

Personen mit niedrigem Sozialstatus geben signifikant häufiger das Vorhandensein einer RA an als Personen mit mittlerem oder höherem Sozialstatus. Die Lebenszeitprävalenz der RA wird weder von der Region noch durch die Gemeindegröße beeinflusst.

\section{Osteoporose}

Eine Übersicht über die Ergebnisse zur Lebenszeitprävalenz der Osteoporose für Befragte ab 50 Jahren, gegliedert nach Geschlecht, Altersgruppe und Sozialstatus, findet sich in - Tab. 3. 8,5\% aller Befragten zwischen 50 und 79 Jahren geben an, jemals eine ärztlich diagnostizierte Osteoporose gehabt zu haben. Frauen geben häufiger an, von einer Osteoporose betroffen zu sein $(13,1 \%)$ als Männer
(3,2\%). Bei Frauen steigt die Lebenszeitprävalenz mit zunehmendem Alter signifikant von 4,1\% in der Altersgruppe der 50 - bis 59-Jährigen auf $25,2 \%$ bei den 70 bis 79-Jährigen. Bei Männern zeigt sich kein derartig signifikanter Anstieg. Der Zusammenhang der Osteoporose mit dem Sozialstatus ist ebenfalls nur bei den weiblichen Befragten ersichtlich: Frauen mit niedrigem Sozialstatus weisen höhere Raten an Osteoporose auf $(18,5 \%)$ als Frauen mit mittlerem (12,0\%) und höherem Sozialstatus $(8,4 \%)$.

Weder bei Frauen noch bei Männern zeigt sich ein Einfluss der Region oder der Gemeindegröße auf die Lebenszeitprävalenz der Osteoporose.

Im Vergleich mit den Ergebnissen des BGS98 zeigt sich, dass sich bei Männern die Prävalenz der Osteoporose in der Altersgruppe der 50- bis 64-Jährigen nicht verändert hat. Bei den 65- bis 79-jähri- gen Männern hat sie zugenommen, liegt aber weiterhin im Bereich zwischen 3 und 4\%. Bei den Frauen liegt ein deutlicher Rückgang in der Altersgruppe der 50- bis 64-Jährigen von 10,4 auf $6,0 \%$ vor. In der Altersgruppe der 65- bis 79-Jährigen hingegen steigt der Anteil von 20,4 auf 23,3\%.

\section{Prävalenz von gleichzeitig bestehenden MSKE}

33,9\% der Männer und 50,1\% der Frauen zwischen 50 und 79 Jahren nennen mindestens eine der 3 genannten MSKE.

- Abb. 2 zeigt, in welchem Maße die Befragten ab dem Alter von 50 Jahren von Doppel- oder Mehrfacherkrankungen betroffen sind.

Arthrose in Kombination mit einer der beiden anderen Erkrankungen weisen insgesamt 8,2\% der Frauen und 3,4\% der Männer auf. Von den Personen, die 


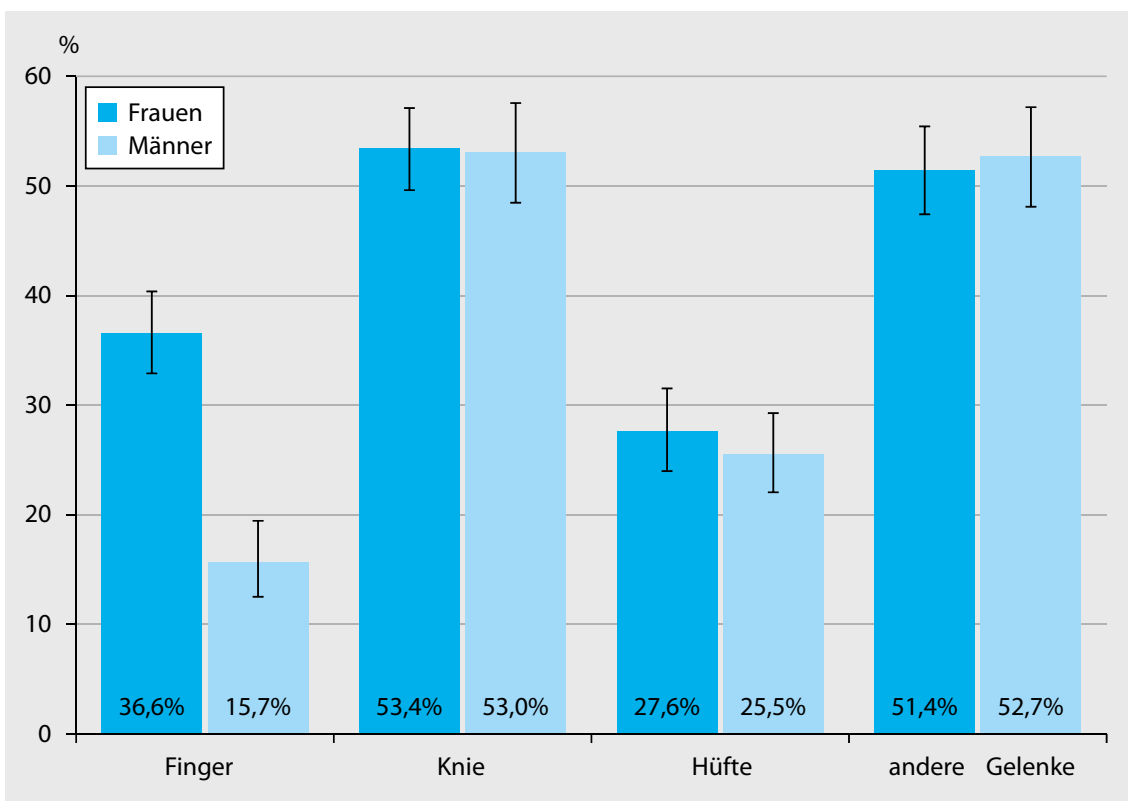

Abb. $1 \Delta$ Lokalisation der Arthrose nach Geschlecht $(n=1746)$

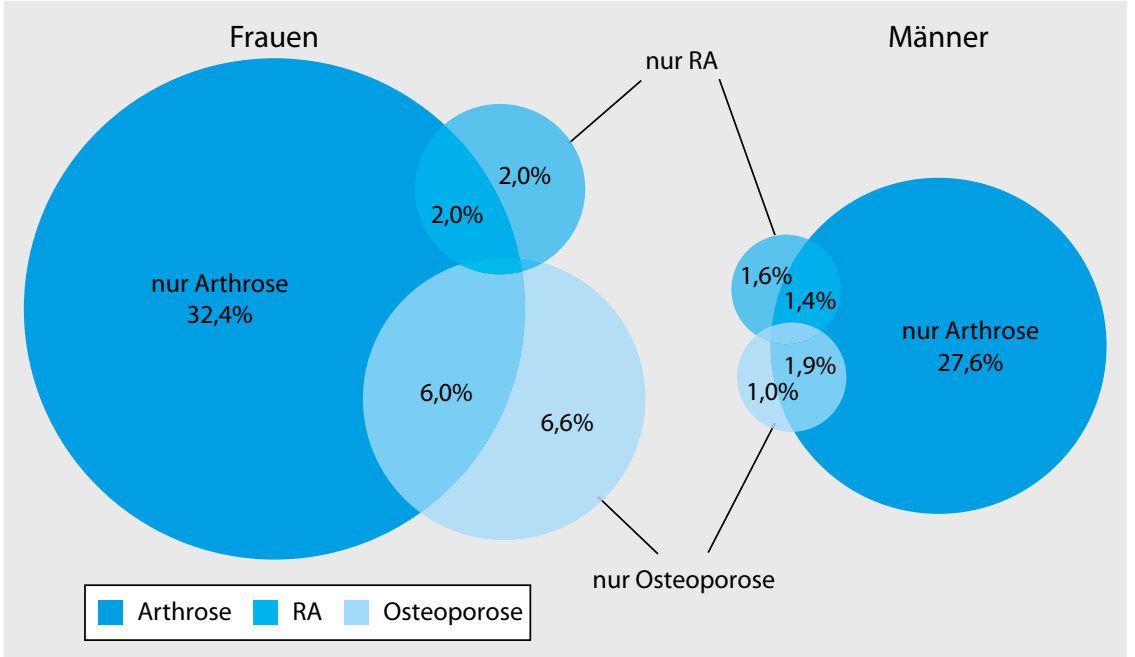

Abb. 2 - Prävalenzschätzung zum gleichzeitigen Auftreten von Arthrose, rheumatoider Arthritis (RA) und Osteoporose für Personen ab 50 Jahren nach Geschlecht, $n=4240$. Gewichtete Fallzahlen, nur Werte ab $1 \%$ benannt

eine RA angeben, gibt die Hälfte gleichzeitig an, an Arthrose zu leiden. Die Hälfte der Frauen sowie zwei Drittel der Männer, die von Osteoporose betroffen sind, berichten, ebenfalls Arthrose zu haben. Das gleichzeitige Auftreten von allen 3 Erkrankungen, liegt unter $1 \%$.

Werden nur die Personen betrachtet, die eine Osteoporose angegeben hatten, dann zeigt sich, dass sich bei diesem Personenkreis eine Assoziation mit RA zeigt (nicht dargestellt).

Die Ergebnisse aus DEGS1 zeigen, dass Arthrose, RA und Osteoporose bei älteren Personen weit verbreitet sind und bei Frauen und Männern unterschiedlich häufig vorkommen. Bei der Einordnung der Prävalenzschätzungen kommt es zu einer Reihe von Einschränkungen. So wird der Terminus "Arthritis“ im englischen Sprachgebrauch als Oberbegriff für alle Gelenkerkrankungen verwendet und kungen ein. Aus diesem Grund ist in englischsprachigen Publikationen zum Oberthema „Arthritis" nicht immer zu trennen zwischen Arthrose, RA, Gicht, systemischem Lupus erythematodes oder Fibromyalgie, was die Differenzierung zwischen degenerativen und entzündlichen Gelenkerkrankungen und damit eine $\mathrm{Zu}$ ordnung der Schätzungen erschwert. Im deutschen Sprachraum bezieht sich „Arthritis“ in erster Linie auf entzündliche Gelenkerkrankungen.

Bei allen MSKE ist darüber hinaus beim Vergleich der in DEGS1 ermittelten Prävalenzen mit denen aus anderen Studien zu berücksichtigen, dass unterschiedliche Altersgruppen, Einschlussund Diagnosekriterien zugrunde liegen können, die keine direkte Gegenüberstellung der Schätzungen zulassen. So variieren z. B. in Studien zu Arthrose die Angaben beträchtlich, je nachdem ob sie auf selbst berichteten oder symptomatischen Angaben oder auf röntgenologischen Befunden basieren. Soweit vorhanden, werden im Folgenden aus Gründen der Vergleichbarkeit in erster Linie Prävalenzschätzungen aus Studien herangezogen, die auf selbst berichteten ärztlichen Diagnosen basieren. Durch den Bezug auf die ärztliche Diagnose ist sichergestellt, dass es sich nicht um reine Selbsteinschätzungen der MSKE handelt und somit z. B. Gelenkbeschwerden ohne ärztlichen Befund nicht eingeschlossen werden. Studien, die auf klinischen oder radiologischen Diagnosekriterien beruhen, zeigen dennoch zum Teil differierende Ergebnisse, auf die Unterschiede wird in den jeweiligen Absätzen eingegangen.

Aufgrund der unterschiedlichen Frageformulierungen für Arthrose ${ }^{1}$ und $\mathrm{Ar}$ thritis ${ }^{2}$ im BGS98 und DEGS1 sind Trendanalysen für diese beiden Erkrankungen nicht möglich. Die Krankheitsabfragen schließt sowohl degenerative („Osteoarthritis") als auch entzündliche Erkran-
1 BGS98: Gelenkverschleiß, Arthrose der Hüftoder Kniegelenke bzw. der Wirbelsäule; DEGS1: Arthrose oder degenerative Gelenkerkrankung.

2 BGS98: Entzündliche Gelenk- oder Wirbelsäulenerkrankung (z. B. chronische Polyarthritis, Rheumatoidarthritis, Morbus Bechterew); DEGS1: rheumatoide Arthritis (ggf. mit Erläuterung „eine schwere entzündliche Gelenkerkrankung, die meist beidseitig auftritt und fast immer die Fingergelenke mit einschließt"). 
Tab. 2 Lebenszeitprävalenz der rheumatoiden Arthritis nach Geschlecht, Altersgruppe und sozialem Status

Altersgruppe

\begin{tabular}{|c|c|c|c|c|c|c|c|c|c|c|}
\hline & & & & & & & & & \\
\hline & & $\begin{array}{l}18 \text { bis } \\
29 \text { Jahre }\end{array}$ & $\begin{array}{l}30 \text { bis } \\
39 \text { Jahre }\end{array}$ & $\begin{array}{l}40 \text { bis } \\
49 \text { Jahre }\end{array}$ & $\begin{array}{l}50 \text { bis } 59 \text { Jah- } \\
\text { re }\end{array}$ & $\begin{array}{l}60 \text { bis } \\
69 \text { Jahre }\end{array}$ & $\begin{array}{l}70 \text { bis } \\
79 \text { Jahre }\end{array}$ & Gesamt & Ja & Nein \\
\hline \multicolumn{11}{|l|}{ Frauen } \\
\hline \multirow[t]{8}{*}{ Sozialstatus } & Niedrig & $2,5 \%$ & & $8,6 \%$ & $6,9 \%$ & $9,1 \%$ & $5,3 \%$ & $5,5 \%$ & 33 & 614 \\
\hline & $95 \%-K I$ & $0,6-9,9 \%$ & & $3,3-20,5 \%$ & $3,0-14,9 \%$ & $4,4-18,0 \%$ & $2,6-10,2 \%$ & $3,6-8,4 \%$ & & \\
\hline & Mittel & $1,8 \%$ & $1,1 \%$ & $1,7 \%$ & $4,1 \%$ & $3,6 \%$ & $4,5 \%$ & $2,7 \%$ & 78 & 2450 \\
\hline & 95\%-KI & $0,8-4,1 \%$ & $0,3-3,9 \%$ & $0,8-3,5 \%$ & $2,5-6,8 \%$ & $2,2-5,9 \%$ & $2,8-7,2 \%$ & $2,1-3,5 \%$ & & \\
\hline & Hoch & & & $1,6 \%$ & $3,2 \%$ & $4,0 \%$ & $4,1 \%$ & $1,8 \%$ & 22 & 862 \\
\hline & 95\%-KI & & & $0,5-5,4 \%$ & $1,5-6,5 \%$ & $1,7-8,8 \%$ & $1,5-10,3 \%$ & $1,1-2,9 \%$ & & \\
\hline & Gesamt & $1,7 \%$ & $0,6 \%$ & $2,9 \%$ & $4,6 \%$ & $4,9 \%$ & $4,9 \%$ & $3,2 \%$ & 133 & 3926 \\
\hline & 95\%-KI & $0,8-3,5 \%$ & $0,2-2,3 \%$ & $1,7-5,0 \%$ & $3,2-6,7 \%$ & $3,3-7,3 \%$ & $3,4-6,9 \%$ & $2,6-4,0 \%$ & & \\
\hline \multicolumn{11}{|l|}{ Männer } \\
\hline \multirow[t]{8}{*}{ Sozialstatus } & Niedrig & $1,9 \%$ & $4,2 \%$ & $3,2 \%$ & $2,4 \%$ & $2,1 \%$ & $12,5 \%$ & $4,0 \%$ & 24 & 562 \\
\hline & 95\%-KI & $0,3-12,2 \%$ & $0,7-20,3 \%$ & $0,7-12,8 \%$ & $0,6-8,8 \%$ & $0,8-5,9 \%$ & $5,4-26,4 \%$ & $2,3-6,8 \%$ & & \\
\hline & Mittel & & $0,5 \%$ & $0,5 \%$ & $1,7 \%$ & $2,8 \%$ & $4,1 \%$ & $1,3 \%$ & 32 & 2064 \\
\hline & 95\%-KI & & $0,1-2,4 \%$ & $0,1-1,5 \%$ & $0,7-4,0 \%$ & $1,6-4,9 \%$ & $1,9-8,7 \%$ & $0,9-1,9 \%$ & & \\
\hline & Hoch & $0,5 \%$ & & $0,6 \%$ & $1,9 \%$ & $3,7 \%$ & $2,0 \%$ & $1,3 \%$ & 15 & 1001 \\
\hline & 95\%-KI & $0,1-3,5 \%$ & & $0,2-2,1 \%$ & $0,6-5,9 \%$ & $1,1-11,6 \%$ & $0,5-7,3 \%$ & $0,7-2,5 \%$ & & \\
\hline & Gesamt & $0,5 \%$ & $1,0 \%$ & $0,9 \%$ & $2,0 \%$ & $2,9 \%$ & $5,8 \%$ & $1,9 \%$ & 71 & 3627 \\
\hline & (95\%-KI) & $0,1-2,6 \%$ & $0,3-3,4 \%$ & $0,4-2,4 \%$ & $1,1-3,6 \%$ & $1,8-4,7 \%$ & $3,4-9,6 \%$ & $1,4-2,5 \%$ & & \\
\hline \multicolumn{11}{|l|}{ Gesamt } \\
\hline \multirow[t]{8}{*}{ Sozialstatus } & Niedrig & $2,2 \%$ & $2,4 \%$ & $5,7 \%$ & $4,4 \%$ & $6,0 \%$ & $7,8 \%$ & $4,8 \%$ & 57 & 1176 \\
\hline & 95\%-KI & $0,7-6,8 \%$ & $0,4-12,5 \%$ & $2,6-12,1 \%$ & $2,2-8,6 \%$ & $3,2-11,1 \%$ & $4,5-13,3 \%$ & $3,4-6,6 \%$ & & \\
\hline & Mittel & $0,8 \%$ & $0,8 \%$ & $1,1 \%$ & $2,9 \%$ & $3,2 \%$ & $4,3 \%$ & $2,0 \%$ & 110 & 4514 \\
\hline & 95\%-KI & $0,4-2,0 \%$ & $0,3-2,2 \%$ & $0,6-2,1 \%$ & $1,8-4,6 \%$ & $2,3-4,6 \%$ & $2,8-6,5 \%$ & $1,6-2,6 \%$ & & \\
\hline & Hoch & $0,3 \%$ & & $1,0 \%$ & $2,5 \%$ & $3,8 \%$ & $2,8 \%$ & $1,5 \%$ & 37 & 1863 \\
\hline & 95\%-KI & $0,0-1,8 \%$ & & $0,4-2,6 \%$ & $1,4-4,6 \%$ & $1,7-8,1 \%$ & $1,2-6,0 \%$ & $1,0-2,4 \%$ & & \\
\hline & Gesamt & $1,1 \%$ & $0,8 \%$ & $1,9 \%$ & $3,3 \%$ & $3,9 \%$ & $5,3 \%$ & $2,5 \%$ & 204 & 7553 \\
\hline & $95 \%-\mathrm{KI}$ & $0,5-2,1 \%$ & $0,3-2,0 \%$ & $1,2-3,0 \%$ & $2,4-4,6 \%$ & $2,9-5,3 \%$ & $3,9-7,1 \%$ & $2,1-3,0 \%$ & & \\
\hline
\end{tabular}

im ärztlichen Interview im BGS98 erfassten ein breiteres Spektrum an Erkrankungen, die in DEGS1 spezifischer formuliert wurden. So wird beispielsweise nach der einführenden Frage nach Arthrose erfasst, welche Gelenke (Finger, Knie, Hüfte, andere) betroffen sind.

\section{Arthrose}

Die Ergebnisse der ersten Welle des europäischen Gesundheitssurveys (European Health Interview Surveys, EHIS) [41] aus 7 Ländern machen deutlich, dass es eine breite Variation der Prävalenz der selbst berichteten ärztlich diagnostizierten Arthrose gibt [3]. Sie reicht von unter 5\% in Rumänien bis etwa 25\% in Ungarn. Darüber hinaus belegen die Ergebnisse, dass in allen Ländern Frauen häufiger eine ärztlich diagnostizierte Arthrose angeben als Männer [3]. In allen Studien ist der Ein- fluss des Alters auf das Auftreten der Arthrose sichtbar. Die Häufigkeit der selbst berichteten Arthrose in Deutschland liegt in verschiedenen Studien bei etwa 20$25 \%$ [42], die in DEGS1 ermittelte Gesamtprävalenz liegt bei $20,2 \%$ und damit innerhalb der beschriebenen Spannweite. Ein Vergleich mit den Daten des Surveys „Gesundheit in Deutschland aktuell“ 2010 $(\text { GEDA10 })^{3}$ des Robert Koch-Instituts zeigt, dass die Lebenszeitprävalenz der Arthrose in DEGS1 bei den 18- bis 79-jährigen Frauen mit 22,5\% etwas niedriger liegt als in GEDA 2010 mit 25,6\%. Bei Männern liegt sie mit 18,0\% geringfügig höher als in GEDA10 (17,3\%). Dies kann der unterschiedlichen Erhebungsmethodik (Face-to-face vs. telefonisch) oder der anderen Stichprobenzusammensetzung

\footnotetext{
3 Eigene Berechnungen für die Altersgruppe der 18 bis 79-Jährigen.
}

geschuldet sein. Bevölkerungsbezogene Prävalenzschätzungen, die auf klinischen oder radiologischen Diagnosestellungen beruhen, liegen für Deutschland nur eingeschränkt vor. In einer Metaanalyse berichten Spahn et al. [14] eine Prävalenz der radiologisch nachweisbaren Gonarthrose von 32,6\% bei Frauen und 24,3\% bei Männern. Die im Vergleich mit den DEGS1Ergebnissen etwas höheren Prävalenzen erklären sich vermutlich dadurch, dass Arthrose-typische Zeichen im Röntgenbild häufig schon ersichtlich sind, ohne dass Schmerzen bei den Betroffenen vorliegen [16].

Die erhöhte Arthroseprävalenz bei Frauen mit niedrigerem Bildungsstatus kann möglicherweise auf den Zusammenhang beruflich bedingter schwerer körperlicher Belastungen auf die ArthroseEntstehung hinweisen [42]. Darüber hinaus spielt die bekannte höhere Adiposi- 
Tab. 3 Lebenszeitprävalenz der Osteoporose nach Geschlecht, Altersgruppe und sozialem Status, nur Befragte ab 50 Jahren

\begin{tabular}{|c|c|c|c|c|c|c|c|}
\hline & & Altersgruppe & & & & Unge & tete Fallzahlen Osteoporose \\
\hline & & 50 bis 59 Jahre & 60 bis 69 Jahre & 70 bis 79 Jahre & Gesamt & Ja & Nein \\
\hline Frauen & & & & & & & \\
\hline Sozialstatus & Niedrig & $7,4 \%$ & $11,5 \%$ & $29,9 \%$ & $18,5 \%$ & 65 & 321 \\
\hline & $95 \%-K I$ & $3,3-15,6 \%$ & $6,8-18,8 \%$ & $22,0-39,3 \%$ & $14,2-23,7 \%$ & & \\
\hline & Mittel & $3,5 \%$ & $13,2 \%$ & $22,7 \%$ & $12,0 \%$ & 178 & 1199 \\
\hline & $95 \%-K I$ & $2,1-5,7 \%$ & $9,9-17,5 \%$ & $18,0-28,2 \%$ & $10,0-14,4 \%$ & & \\
\hline & Hoch & $3,3 \%$ & $12,0 \%$ & $19,7 \%$ & $8,4 \%$ & 41 & 389 \\
\hline & $95 \%-K I$ & $1,3-8,1 \%$ & $7,0-19,8 \%$ & $11,3-32,0 \%$ & $6,1-11,6 \%$ & & \\
\hline & Gesamt & $4,1 \%$ & $12,7 \%$ & $25,2 \%$ & $13,1 \%$ & 284 & 1909 \\
\hline & 95\%-KI & $2,7-6,0 \%$ & $10,2-15,6 \%$ & $21,2-29,8 \%$ & $11,3-15,1 \%$ & & \\
\hline Männer & & & & & & & \\
\hline Sozialstatus & Niedrig & $4,3 \%$ & $2,8 \%$ & $3,3 \%$ & $3,6 \%$ & 11 & 312 \\
\hline & $95 \%-K I$ & $1,3-13,3 \%$ & $0,8-8,9 \%$ & $0,8-11,9 \%$ & $1,7-7,3 \%$ & & \\
\hline & Mittel & $3,5 \%$ & $2,7 \%$ & $3,5 \%$ & $3,3 \%$ & 46 & 1066 \\
\hline & 95\%-KI & $1,9-6,2 \%$ & $1,5-5,0 \%$ & $1,9-6,7 \%$ & $2,3-4,6 \%$ & & \\
\hline & Hoch & $1,9 \%$ & $1,9 \%$ & $5,3 \%$ & $2,6 \%$ & 13 & 559 \\
\hline & 95\%-KI & $0,5-7,0 \%$ & $0,7-4,9 \%$ & $1,6-16,2 \%$ & $1,3-5,0 \%$ & & \\
\hline & Gesamt & $3,3 \%$ & $2,6 \%$ & $3,8 \%$ & $3,2 \%$ & 70 & 1937 \\
\hline & $95 \%-\mathrm{KI}$ & $2,0-5,3 \%$ & $1,6-4,1 \%$ & $2,3-6,3 \%$ & $2,4-4,2 \%$ & & \\
\hline Gesamt & & & & & & & \\
\hline Sozialstatus & Niedrig & $5,7 \%$ & $7,7 \%$ & $20,6 \%$ & $11,9 \%$ & 76 & 633 \\
\hline & $95 \%-\mathrm{KI}$ & $2,9-10,9 \%$ & $4,8-12,0 \%$ & $15,2-27,2 \%$ & $9,4-15,1 \%$ & & \\
\hline & Mittel & $3,5 \%$ & $8,4 \%$ & $13,9 \%$ & $7,9 \%$ & 224 & 2265 \\
\hline & $95 \%-\mathrm{Kl}$ & $2,3-5,1 \%$ & $6,4-10,9 \%$ & $11,0-17,3 \%$ & $6,7-9,3 \%$ & & \\
\hline & Hoch & $2,6 \%$ & $5,7 \%$ & $10,4 \%$ & $5,0 \%$ & 54 & 948 \\
\hline & 95\%-KI & $1,2-5,4 \%$ & $3,6-8,8 \%$ & $6,3-16,7 \%$ & $3,7-6,9 \%$ & & \\
\hline & Gesamt & $3,8 \%$ & $7,6 \%$ & $16,1 \%$ & $8,5 \%$ & 354 & 3846 \\
\hline & 95\%-KI & $2,7-5,4 \%$ & $6,1-9,6 \%$ & $13,4-19,2 \%$ & $7,3-9,9 \%$ & & \\
\hline
\end{tabular}

tasprävalenz bei Personen mit niedrigem Sozialstatus [43] eine Rolle bezüglich der Arthrosehäufigkeiten in dieser Bevölkerungsgruppe.

\section{Rheumatoide Arthritis (RA)}

Bei einer Einordnung der Prävalenzschätzung ist $\mathrm{zu}$ berücksichtigen, dass diese stark von der formulierten Frage nach der Erkrankung abhängt. Je nachdem, ob nach Arthritis allgemein oder spezifisch nach „rheumatoider Arthritis“ gefragt wird, kommt es zu deutlich unterschiedlichen Schätzungen. Aus diesem Grund wurde in DEGS1 explizit nach „rheumatoider Arthritis“ gefragt; nichtsdestotrotz lassen sich Missklassifikationen durch die Befragten nicht ausschließen. Der German Rheumatoid Arthritis Population Survey (GRAPS) des Deutschen Rheumaforschungszentrums (DRFZ) zeigt, dass Personen, die eine kli- nisch gesicherte RA haben, diese bei der schriftlichen Befragung zu rund 70\% der Fälle korrekt angeben, Personen mit anderen Gelenkerkrankungen jedoch sehr häufig fälschlicherweise eine RA-Diagnose berichten [44]. Es kann davon ausgegangen werden, dass in DEGS1 aufgrund der Fragestellung damit zu rechnen ist, dass die Prävalenz für chronische entzündliche und rheumatische Gelenkerkrankungen überschätzt wird.

Die in DEGS1 für die Altersgruppe der 18- bis 44-Jährigen beobachteten Prävalenzschätzungen zu RA entsprechen den Einschätzungen zur Prävalenz der selbst berichteten RA aus GRAPS, für die anderen Altersgruppen liegen sie niedriger [45]. In GEDA10 berichten 7,1\% der Frauen und 4,1\% der Männer, dass bei ihnen jemals eine „Arthritis, rheumatische Arthritis oder chronische Polyarthritis ärztlich festgestellt" wurde (Lebenszeitprävalenz). Insgesamt fallen sowohl die
Schätzungen aus GEDA10 als auch aus DEGS1 deutlich höher aus als Schätzungen aus bevölkerungsbezogenen Untersuchungen zur RA-Prävalenz mit spezifischen Informationen zur Diagnosestellung [3, 46, 47]. Wasmus et al. [48] schätzen, basierend auf den Klassifikationskriterien der RA [28], die Häufigkeit der RA in Deutschland auf 0,5-0,8\% der erwachsenen Bevölkerung. Die so ermittelten Prävalenzen fallen niedriger aus als die Prävalenzschätzungen, die auf Selbstberichten beruhen. Möglicherweise sind Fehlklassifikationen durch die Befragten die Erklärung hierfür.

Eine Reihe von Studien zeigt den $\mathrm{Zu}$ sammenhang zwischen dem Vorliegen einer RA und niedrigem sozialem Status auf $[49,50]$. Die in DEGS gefundenen höheren Prävalenzen bei Männern entsprechen diesen Ergebnissen. 


\section{Osteoporose}

Wie bei Arthrose und RA unterscheiden sich Prävalenzschätzungen zur Osteoporose nach Art und Quelle der Datenerhebung und Zusammensetzung der Studienpopulation. Internationale Prävalenzschätzungen zur Osteoporose basieren außerdem häufig auf der Ermittlung einer niedrigen Knochendichte, die in einem bevölkerungsbezogenen Survey wie DEGS1 nicht ermittelt werden kann. Diese Prävalenzschätzungen sind aufgrund der unterschiedlichen Methodik nicht mit denen des DEGS1 vergleichbar.

Der in DEGS1 gefundene Alters- und Geschlechterunterschied bei Osteoporose zeigt sich in verschiedenen Studien in Deutschland. Meisinger et al. [51] geben in der Augsburger MONICA-Studie die Lebenszeitprävalenz der selbst berichteten Osteoporose in der Altersgruppe der 25- bis 74-Jährigen auf der Grundlage der Datenerhebung 1994/1995 mit 7\% bei Frauen und 1\% bei Männern an. Anhand von Routinedaten der Gmünder Ersatzkasse (Diagnosen und Arzneimittelverordnungen) kommen Häussler et al. [52] zu einer Osteoporoseprävalenz von 23,3\% bei Frauen und 7,1\% bei Männern in der Altersgruppe der der 50- bis 64-Jährigen und $46,7 \%$ bei Frauen bzw. 11,4\% bei Männern in der Altersgruppe der 65- bis 74-Jährigen.

Aus verschiedenen weiteren Surveys des RKI liegen Angaben zur ärztlich diagnostizierten Osteoporose vor. Im telefonischen Survey 2003 lag die Lebenszeitprävalenz bei Frauen ab 45 Jahren bei 14,2\% $[11]^{4}$, in GEDA10 (Befragte ab 50 Jahre) betrug sie $15,7 \%$ bei Frauen und 4,1\% bei Männern. Die Prävalenz stieg bei Frauen signifikant an; von $7,6 \%$ bei den 50 bis 59 -Jährigen auf $23,7 \%$ bei den 70 - bis 79-Jährigen. Bei Männern war keine solche Altersabhängigkeit zu beobachten. In der EVOS-Studie liegt die Prävalenz einer klinisch diagnostizierten manifesten Osteoporose bei 60- bis 69-jährigen Frauen bei $13,0 \%$, bei 70- bis 79-Jährigen bei 22,8\%, bei Männern bei 5,1 bzw. 5,8\% [53], die in DEGS1 ermittelten Ergebnisse ent-

\footnotetext{
${ }^{4}$ Osteoporose wurde hier nur bei Frauen erfragt.
}

sprechen trotz unterschiedlicher Methodik diesen Ergebnissen.

Bei nationalen Vergleichen ist zu berücksichtigen, dass sich in Deutschland die Leitlinienempfehlungen und Abrechnungsmodalitäten in den letzten Jahren verändert haben [29]. Daher ist die Berücksichtigung des Erhebungszeitraums für Vergleiche von besonderer Bedeutung. Bei den 50- bis 65-jährigen Frauen hat sich eine Verringerung der Osteoporoseraten in DEGS1 im Vergleich zum BGS98 gezeigt. Diese ist möglicherweise darauf zurückzuführen, dass Knochendichtemessungen nach aktuellen Leitlinienempfehlungen bei jüngeren Personen weniger häufig durchgeführt werden, sodass eine Diagnosestellung ausbleibt bzw. erst in späteren Jahren aufgrund einer sich manifestierenden Osteoporose erfolgt.

\section{Fazit und Ausblick}

MSKE spielen besonders für die ältere und alte Bevölkerung in Deutschland eine bedeutende Rolle und die hier vorgestellten Ergebnisse belegen die sozialmedizinische Bedeutsamkeit dieser Erkrankungen. DEGS1 bietet vielfältige Möglichkeiten, MSKE einzeln oder kombiniert zu betrachten und mit relevanten Einflussgrößen wie Inanspruchnahme von medizinischer Versorgung, aktuelle Medikation, der Häufigkeit von künstlichen Gelenken und anderen chronischen Erkrankungen in Zusammenhang zu setzen. Darüber hinaus kann das Wechselspiel mit körperlichen Funktionseinschränkungen beschrieben werden. Auch ist geplant, den bekannten $\mathrm{Zu}$ sammenhang von Übergewicht/Adipositas und dem Vorliegen einer Arthrose zu analysieren. Analysen zu Gelenkschmerzen, einem wesentlichen Symptom von Arthrose und RA, sollen zudem Informationen zu den aktuellen Beeinträchtigungen infolge von MSKE geben.

Für Osteoporose sollen Auswertungen unter Einbezug von Fragilitätsfrakturen und deren Lokalisation durchgeführt werden. Diese Faktoren können Hinweise auf eine verringerte Knochendichte und auf eine vorliegende, nicht bekannte Osteoporose geben. In diesem Zusammenhang sind darüber hinaus Analysen unter
Einbeziehung von Stürzen und Sturzangst geplant.

Insgesamt bietet DEGS1 eine Vielfalt von Möglichkeiten, MSKE in Zusammenhang mit relevanten Einflussgrößen zu setzen und somit den Gesundheitszustand der zu Hause lebenden Bevölkerung präzise zu beschreiben.

\section{Korrespondenzadresse}

\section{Dr. J. Fuchs}

Abteilung für Epidemiologie und Gesundheitsmonitoring, Robert Koch-Institut General-Pape-Str. 62-66, 12101 Berlin FuchsJ@rki.de

Externe Kooperationen. Klinik und Poliklinik für Radiologie und Nuklearmedizin, Zentrum für Muskelund Knochenforschung, Charité Berlin.

Deutsches Rheumaforschungszentrum, Berlin.

Finanzierung der Studie. Die Studie wurde finanziert mit Mitteln des Robert Koch-Instituts und des Bundesministeriums für Gesundheit.

Interessenkonflikt. Die korrespondierende Autorin gibt für sich und ihre Koautoren an, dass kein Interessenkonflikt besteht.

\section{Literatur}

1. Mody GM, Brooks PM (2012) Improving musculoskeletal health: global issues. Best Pract Res Clin Rheumatol 26:237-249

2. Choong P, Brooks P (2012) Achievements during the bone and joint decade 2000-2010. Best Pract Res Clin Rheumatol 26:173-181

3. Woolf AD, Erwin J, March L (2012) The need to address the burden of musculoskeletal conditions. Best Pract Res Clin Rheumatol 26:183-224

4. World Health Organisation (WHO) (2003) The burden of musculoskeletal conditions at the start of the new millennium. Report of a WHO Scientific Group Technical Report Series. Geneva. 919

5. Fuchs J, Busch M, Lange C, Scheidt-Nave C (2012) Prevalence and patterns of morbidity among adults in Germany. Results of the German telephone health interview survey German Health Update (GEDA) 2009. Bundesgesundheitsbl Gesundheitsforsch Gesundheitsschutz 55:576-586

6. Merkesdal S, Huelsemann JL, Mittendorf T et al (2006) Produktivitätskosten der rheumatoiden Arthritis in Deutschland. Kostenzusammensetzung und Prädiktion der Hauptkostenkomponenten. Z Rheumatol 65:527-534

7. Haussler B, Gothe H, Gol D et al (2007) Epidemiology, treatment and costs of osteoporosis in Germany - the BoneEVA Study. Osteoporos Int 18:77-84

8. Gunther KP, Sturmer T, Sauerland S et al (1998) Prevalence of generalised osteoarthritis in patients with advanced hip and knee osteoarthritis: the Ulm Osteoarthritis Study. Ann Rheum Dis 57:717723

9. Michael JW, Schluter-Brust KU, Eysel P (2010) The epidemiology, etiology, diagnosis, and treatment of osteoarthritis of the knee. Dtsch Arztebl Int 107:152-162 
10. Rosemann T, Laux G, Kuehlein T (2007) Osteoarthritis and functional disability: results of a cross sectional study among primary care patients in Germany. BMC Musculoskelet Disord 8:79

11. Scheidt-Nave C, Starker A (2005) Osteoporoseprävalenz und assoziierte Versorgungsmuster bei Frauen im Alter ab 45 Jahren in Deutschland. Ergebnisse des ersten telefonischen Gesundheitssurveys 2003. Bundesgesundheitsbl Gesundheitsforsch Gesundheitsschutz 48:1338-1347

12. Scheidt-Nave C, Ziegler R, Raspe H (1998) Epidemiologie der Osteoporose. Med Klin (Munich) 93(Suppl 2):7-11

13. Schneider S, Schmitt G, Richter W (2006) Prevalence and correlates of inflammatory arthritis in Germany: data from the First National Health Survey. Rheumatol Int 27:29-38

14. Spahn G, Schiele R, Hofmann GO et al (2011) Die Prävalenz der radiologischen Gonarthrose in Bezug zu Lebensalter, Geschlecht, Jahrgangskohorte und ethnischer Zugehörigkeit. Eine Metaanalyse. Z Orthop Unfall 149:145-152

15. Sun Y, Sturmer T, Gunther KP, Brenner H (1997) Inzidenz und Prävalenz der Cox- und Gonarthrose in der Allgemeinbevölkerung. Z Orthop Ihre Grenzgeb 135:184-192

16. Thiem U, Schumacher J, Zacher J et al (2008) Prävalenz von muskuloskelettalen Beschwerden und selbstberichteter Gelenkarthrose in der Herner Bevölkerung: Ein Telefon-Survey. Z Rheumatol 67:432-439

17. Zeidler H, Merkesdal S, Hulsemann JL (2003) Early arthritis and rheumatoid arthritis in Germany. Clin Exp Rheumatol 21:106-112

18. Woolf AD, Pfleger B (2003) Burden of major musculoskeletal conditions. Bull World Health Organ 81:646-656

19. Felson DT, Lawrence RC, Dieppe PA et al (2000) Osteoarthritis: new insights. Part 1: the disease and its risk factors. Ann Intern Med 133:635-646

20. Blagojevic M, Jinks C, Jeffery A, Jordan KP (2010) Risk factors for onset of osteoarthritis of the knee in older adults: a systematic review and meta-analysis. Osteoarthritis Cartilage 18:24-33

21. Swift A (2012) Osteoarthritis 1: physiology, risk factors and causes of pain. Nurs Times 108:12-15

22. Muthuri SG, Hui M, Doherty M, Zhang W (2011) What if we prevent obesity? Risk reduction in knee osteoarthritis estimated through a meta-analysis of observational studies. Arthritis Care Res (Hoboken) 63:982-990

23. Oliver JE, Silman AJ (2009) What epidemiology has told us about risk factors and aetiopathogenesis in rheumatic diseases. Arthritis Res Ther 11:223

24. Raychaudhuri S (2010) Recent advances in the genetics of rheumatoid arthritis. Curr Opin Rheumatol 22:109-118

25. Robert Koch Institut (RKI) (2010) Entzündlichrheumatische Erkrankungen. Gesundheitsberichterstattung des Bundes, Heft 49. RKI, Berlin

26. Sangha O (2000) Epidemiology of rheumatic diseases. Rheumatology (Oxford) 39(Suppl 2):3-12

27. Wollenhaupt J, Krüger K (2012) Frühe und fortgeschrittene rheumatoide Arthritis. Z Rheumatol 71:53-63

28. Aletaha D, Neogi T, Silman AJ et al (2010) 2010 rheumatoid arthritis classification criteria: an American College of Rheumatology/European League Against Rheumatism collaborative initiative. Ann Rheum Dis 69:1580-1588

29. Dachverband Osteologie (DVO) (2009) Leitlinie 2009 zur Prophylaxe, Diagnostik und Therapie der Osteoporose bei Erwachsenen. Langfassung. Osteologie 4:304-324
30. World Health Organisation (WHO) (1994) Assessment of fracture risk and its application to screening for postmenopausal osteopororsis. Technical report series. World Health Organization, Geneva, $\mathrm{S} 843$

31. Endres HG, Dasch B, Lungenhausen M et al (2006) Patients with femoral or distal forearm fracture in Germany: a prospective observational study on health care situation and outcome. BMC Public Health 6:87

32. Schumacher J, Thiem U, Smektala R et al (2007) Osteoporose und Sturz: medizinische Versorgung älterer Menschen mit einem erhöhten Frakturrisiko in Deutschland. Z Arztl Fortbild Qualitatssich 101:593-597

33. Gößwald A, Lange M, Kamtsiuris P, Kurth BM (2012) DEGS: Studie zur Gesundheit Erwachsener in Deutschland. Bundesweite Quer- und Längsschnittstudie im Rahmen des Gesundheitsmonitorings des Robert Koch-Instituts. Bundesgesundheitsbl Gesundheitsforsch Gesundheitsschutz 55:775-780

34. Kamtsiuris $P$, Lange $M$, Hoffmann R et al (2013) Die erste Welle der Studie zur Gesundheit Erwachsener in Deutschland (DEGS1). Stichprobendesign, Response, Gewichtung und Repräsentativität. Bundesgesundheitsbl Gesundheitsforsch Gesundheitsschutz 56:620-630

35. Kurth BM (2012) Das RKI-Gesundheitsmonitoring - was es enthält und wie es genutzt werden kann. Public Health Forum 20(76):4.e1-4.e3

36. Kurth BM, Lange C, Kamtsiuris P, Hölling H (2009) Gesundheitsmonitoring am Robert Koch-Institut. Sachstand und Perspektiven. Bundesgesundheitsbl Gesundheitsforsch Gesundheitsschutz 52:557-570

37. Scheidt-Nave C, Kamtsiuris P, Goesswald A et al (2012) German health interview and examination survey for Adults (DEGS) - design, objectives and implementation of the first data collection wave. BMC Public Health 12:730

38. Gößwald A, Lange M, Dölle R, Hölling H (2013) Die erste Welle der Studie zur Gesundheit Erwachsener in Deutschland (DEGS1). Gewinnung von Studienteilnehmenden, Durchführung der Feldarbeit und Qualitätsmanagement. Bundesgesundheitsbl Gesundheitsforsch Gesundheitsschutz 56:611-619

39. Robert Koch-Institut (Hrsg) (2009) DEGS: Studie zur Gesundheit Erwachsener in Deutschland - Projektbeschreibung. Beiträge zur Gesundheitsberichterstattung des Bundes. RKI, Berlin

40. Lampert T, Kroll L, Müters S, Stolzenberg H (2013) Messung des sozioökonomischen Status in der Studie zur Gesundheit Erwachsener in Deutschland (DEGS1). Bundesgesundheitsbl Gesundheitsforsch Gesundheitsschutz 56:631-636

41. Statistical Office of the European Union (EUROSTAT) European Health Interview Survey (EHIS) collection round 2008. Reference Metadata in Euro SDMX Metadata Structure (ESMS). http://epp.eurostat.ec.europa.eu/cache/ITY_SDDS/EN/hlth_ ehis_esms.htm

42. Schneider S, Schmitt G, Mau H et al (2005) Prävalenz und Korrelate der Osteoarthrose in der BRD. Orthopäde 34:782-790

43. Mensink GBM, Schienkiewitz A, Haftenberger M et al (2013) Übergewicht und Adipositas in Deutschland. Ergebnisse der Studie zur Gesundheit Erwachsener in Deutschland (DEGS1). Bundesgesundheitsbl Gesundheitsforsch Gesundheitsschutz 56:786-794
44. Westhoff G, Schneider M, Raspe H et al (2007) Arthrose oder Arthritis? Wissen Betroffene, was sie meinen? In: 35. Kongress der DGRh und 21. Jahrestagung der ARO, Hamburg, 19.-22. September 2007

45. Westhoff G, Schneider M, Raspe H et al (2009) Advance and unmet need of health care for patients with rheumatoid arthritis in the German population - results from the German Rheumatoid Arthritis Population Survey (GRAPS). Rheumatology 48:650-657

46. Symmons DP (2002) Epidemiology of rheumatoid arthritis: determinants of onset, persistence and outcome. Best Pract Res Clin Rheumatol 16:707722

47. Helmick CG, Felson DT, Lawrence RC et al (2008) Estimates of the prevalence of arthritis and other rheumatic conditions in the United States: part I. Arthritis Rheum 58:15-25

48. Wasmus A, Kindel P, Mattussek S, Raspe HH (1989) Activity and severity of rheumatoid arthritis in Hannover/FRG and in one regional referral center. Scand J Rheumatol Suppl 79:33-44

49. Group ES (2000) Socioeconomic deprivation and rheumatoid disease: what lessons for the health service? Ann Rheum Dis 59:794-799

50. Harrison MJ, Tricker KJ, Davies L et al (2005) The relationship between social deprivation, disease outcome measures, and response to treatment in patients with stable, long-standing rheumatoid arthritis. J Rheumatol 32:2330-2336

51. Meisinger C, Wildner M, Stieber J et al (2002) Epidemiologie der Extremitätenfrakturen. Orthopäde 31:92-99

52. Häussler B, Gothe $H$, Mangiapane S et al (2006) Versorgung von Osteoporose-Patienten in Deutschland: Ergebnisse der BoneEVA-Studie. Dtsch Arztebl 103:2542-2548

53. Leidig-Bruckner G, Limberg B, Felsenberg D et al (2000) Sex difference in the validity of vertebral deformities as an index of prevalent vertebral osteoporotic fractures: a population survey of older men and women. Osteoporos Int 11:102-119 